\title{
Controllability of Formation Systems on Special Orthogonal Groups over Directed Graphs
}

\author{
Michael Sinner,* Student Member, IEEE, Xudong Chen, Member, IEEE, and Lucy Pao, Fellow, IEEE
}

\begin{abstract}
Gradient flows provide a means for a networked formation system to reach and stabilize at a target configuration. However, the decentralization constraints and the geometry of the state space makes the appearance of stable but undesired configurations inevitable. The presence of these undesired stable configurations precludes global convergence to the target configuration. In this paper, we address the issue by considering a controlled formation system on special orthogonal groups over a directed graph. Agents of the system are tasked with stabilizing from others at target relative attitudes. The nominal dynamics of the agents are gradient flows of certain potential functions. These functions are parameter dependent, pretuned by the controller. To prevent the formation system from being trapped at an undesired configuration, we formulate and address the problem of whether the controller can steer the system from any configuration to any other configuration by retuning, on the fly, the parameters of the potential functions. We show that the answer is affirmative provided that the underlying graph is rooted with a single root node being fully actuated. We formulate the result as a main theorem and provide a complete proof of the result.
\end{abstract}

Index Terms-Formation systems, control-linear systems, Lie groups, geometric control

\section{INTRODUCTION}

Gradient descent has been widely used in multi-agent control for guiding a networked system to reach and stabilize at a target configuration. Often, individual agents in a system are assigned with certain potential functions, which depend only on the local information accessible to the agents. The dynamics of each agent are then realized as the gradient flow of the associated potential function. Stabilization at the nominal operation point can be achieved if the potential functions are pre-tuned such that the target configuration is a global (or at least a local) minimum point of the cost function for the entire networked system, defined as the sum of all the potential functions associated with the individual agents.

If a networked system needs to transition between distinct operation points from time to time, then we face the control problem of steering the system from one target configuration to another. Such steering operations can be achieved if we allow the potential functions to depend on certain time-varying tuning parameters, which can also be viewed as reference points for the system. The controller thus steers the networked system by retuning, on the fly, the potential functions for the individual agents. For example, if the retuning is made such that the new target configuration emerges as a global (or

${ }^{*}$ Corresponding author. M. Sinner, X. Chen, and L. Pao are with the Department of Electrical, Computer, and Energy Engineering, University of Colorado Boulder, CO 80309, emails:\{michael.sinner, xudong.chen, lucy.pao\}@colorado.edu local) minimum point of the corresponding cost function, then one would expect that the dynamics of the networked system will converge asymptotically to the new target configuration provided that it is not too far away from the last one.

\section{A. Motivating Example}

To illustrate, we take an example from distance-based formation control [1]-[3]. Consider a networked system of $N$ autonomous agents evolving in an Euclidean space, tasked with stabilizing at target distances from other agents. By convention, we use an undirected graph $G=(V, E)$ to represent the information flow topology of the formation system. Specifically, agents are assigned to nodes $v_{i}$ of $G$. Edges $v_{i} v_{j}$ mean that agents $v_{i}$ and $v_{j}$ can access the relative distance $\left\|x_{j}(t)-x_{i}(t)\right\|$ between them at all times. To stabilize agent $v_{i}$ from its neighbors $v_{j}$ at target distances $\bar{d}_{i j}$, the controller can assign the agent the following potential function:

$$
\xi_{i}\left(x_{1}, \ldots, x_{N}\right):=\frac{1}{4} \sum_{v_{j} \in V_{i}}\left(\left\|x_{j}-x_{i}\right\|^{2}-\bar{d}_{i j}^{2}\right)^{2},
$$

where $V_{i}$ is the collection of neighbors of agent $v_{i}$. The potential function is minimized if the target distances are achieved, i.e., $\left\|x_{j}-x_{i}\right\|=\bar{d}_{i j}$ for all $v_{j} \in V_{i}$. The agent $v_{i}$ implements the following gradient-dynamics for minimizing $\xi_{i}$ :

$$
\dot{x}_{i}=-\frac{\partial \xi_{i}}{\partial x_{i}}=\sum_{v_{j} \in V_{i}}\left(\left\|x_{j}-x_{i}\right\|^{2}-\bar{d}_{i j}^{2}\right)\left(x_{j}-x_{i}\right) .
$$

Local and global convergence of the above dynamics and various variations have been investigated extensively in the literature (see a survey paper [4] and references therein). In the example, the target distances $\bar{d}_{i j}$ are the tuning parameters. By setting the values of $\bar{d}_{i j}$ appropriately, any given configuration can be realized as a global minimum point of the cost function $J:=1 / 2 \sum_{i=1}^{N} \xi_{i}$. Thus, the controller can manage to stabilize the formation system at different target configurations.

However, for the above control scheme to work, a critical assumption is that for a given cost function (which is parameterized by the tuning parameters), all of its local minimum points need to be the target configurations. To see this, we first note that having a target configuration as a local, or even global, minimum point of the cost function guarantees only local convergence. By resetting the target distances and, hence, having the new target configuration to be a local (or global) minimum point of the corresponding cost function does not necessarily make the formation system converge to it unless the previous target configuration lies in the basin of attraction 
of the new one. On the other hand, it is well known that the cost function possesses multiple local minimum points after modulo translations and rotations [3], [5]-[7]. It still remains an open problem to determine when the local minimum points comprise only target configurations. A partial solution was provided in Chen et al. [3]. Similar stabilization problems, but for bearing- or angle-based formation control, can be found, for example, in Zhao and Zelazo [8] and Jing et al. [9]. We also refer the reader to some other relevant problems in formation control, such as formation tracking [10] and formation containment [11].

The approach we take to address the steering problem is to simply allow the tuning parameters to be time-varying during the transition from one target configuration to another, i.e., we treat the tuning parameters as "control inputs." If the resulting system is controllable, then the transition can always be achieved by manipulating, on the fly, the tuning parameters. In the example of distance-based formation control, the associated control system, adapted from the gradient dynamics (1), is given by the following:

$$
\dot{x}_{i}(t)=\sum_{v_{j} \in V_{i}} u_{i j}(t)\left(x_{i}(t)-x_{j}(t)\right), \quad \forall v_{i} \in V,
$$

where we have replaced the tuning parameters $\bar{d}_{i j}^{2}$ with $u_{i j}(t)$ to indicate that they are now treated as control inputs. We allow $u_{i j}(t)$ to take values in $\mathbb{R}$. Note that the drifting term $\sum_{v_{j} \in V_{i}}\left\|x_{j}-x_{i}\right\|^{2}\left(x_{j}-x_{i}\right)$ has been omitted for ease of presentation. We can do this because (2) is a bilinear control system [12]. Specifically, if system (2) is controllable, then so is the system obtained by adding a drifting term to (2). Controllability of bilinear systems of type (2) has been investigated in Chen and Brockett [13] for the case where $G$ is undirected with $u_{i j}(t)=u_{j i}(t)$ being reciprocal, and, in Chen et al. [14] for the case where $G$ is a directed graph. In the latter case, the summation in (2) is over the in-neighbors of $v_{i}$ and the reciprocity $u_{i j}(t)=u_{j i}(t)$ is not required.

\section{B. Formation Control on Special Orthogonal Groups}

The steering problem we consider in the paper is similar to the example given in the previous subsection. But, instead of treating agents as points, we consider agents as rigid bodies, whose states are represented by $3 \times 3$ special orthogonal matrices. Thus, the underlying configuration space is now a Cartesian product of special orthogonal groups. The change of configuration space makes the formation system essentially different from the one that is defined on a Euclidean space. The rigid body formation system is tasked with stabilizing the agents at certain relative attitudes from their neighbors. The dynamics of each agent will again be realized as the gradient flow of a certain potential function that depends on (controlled) tuning parameters. The problem we are concerned with is manipulating the tuning parameters to steer the formation system from one target configuration to another.

Improving our understanding of the controllability of rigid body formations has ramifications for various applications, such as satellite formations. Recent NASA mission examples include GRACE, which measures the Earth's gravitational and magnetic fields [15]; TanDEM-X, which provides imaging and elevation mapping of the Earth's surface [16]; and TROPICS, which measures global pollution and climate change [17]. Satellite formations offer an effective alternative for widefield, distributed data collection missions where single-vehicle solutions would be infeasible. Coordination of satellite formations with appropriate attitudes is critical for enhancing the capability of the overall system to collect high-value data and for extending the lifetime of the system by improving the quality of local state estimations (e.g., to avoid collisions).

We will now provide a precise description of the aforementioned control problem. Let $\mathrm{SO}(3)$ be the special orthogonal group for $\mathbb{R}^{3}$ and $\theta_{i}(t) \in \mathrm{SO}(3)$ be the state of agent $v_{i}$. Note that $\theta_{i}(t)$ is the absolute attitude of agent $v_{i}$ with respect to a global framework. We do not assume that all the agents in the formation system are able to access their own states. Instead, there is only one agent, termed the leader and denoted by $v_{1}$, that can access its state $\theta_{1}(t)$ for all time. The other agents will be referred to as followers, which can only access relative attitudes with respect to their in-neighbors. Specifically, if there is an edge $v_{j} v_{i}$ from $v_{j}$ to $v_{i}$ in $G$ (in the sequel, we will assume that the information flow topology is directed), then agent $v_{i}$ is able to access the following special orthogonal matrix for all $t$ :

$$
\phi_{i j}(t):=\theta_{i}^{\top}(t) \theta_{j}(t) .
$$

We call $\phi_{i j}$ the relative attitude and note that $\theta_{j}=\theta_{i} \phi_{i j}$. Note also that $\phi_{j i}=\phi_{i j}^{\top}$, although $v_{i} v_{j}$ may not be an edge of the directed graph $G$.

To stabilize agents $v_{i}$ from their in-neighbors at relative attitudes, we consider assigning to the agents the following quadratic potential functions:

$$
\xi_{i}\left(\theta_{1}, \ldots, \theta_{N}\right):=\sum_{v_{j} \in V_{i}^{+}}\left\|\theta_{i}^{\top} \theta_{j}-U_{i j}\right\|_{F}^{2},
$$

where $V_{i}^{+}$is the collection of in-neighbors of $v_{i}$ and $\|A\|_{F}:=\sqrt{\operatorname{tr}\left(A A^{\top}\right)}$ is the Frobenius norm of an arbitrary real matrix $A$. The matrix $U_{i j} \in \mathbb{R}^{3 \times 3}$ is a tuning parameter determined by the controller. The nominal gradient dynamics of (4) with respect to the normal metric (see Section II-B) for the agent $v_{i}$ are then given by

$$
\dot{\theta}_{i}(t)=\sum_{v_{j} \in V_{i}^{+}} \theta_{i}(t)\left(\phi_{i j}(t) U_{i j}^{\top}-U_{i j} \phi_{j i}(t)\right) .
$$

Note, in particular, that if each $U_{i j}$ is a scalar multiple of identity, i.e., $U_{i j}=u_{i j} I$ with $u_{i j}$ positive, then (5) is reduced to the "gradient consensus algorithm" [18], the goal of which is to drive all the $\theta_{i}(t)$ to converge to the same value (see, also, the Lohe model [19], [20] on the unitary group as a non-abelian generalization of the Kuramoto model [21]). A similar consensus algorithm, defined on Stiefel manifolds, can be found in the recent work of Markdahl et al. [22]. Note that global convergence to a consensus state is not always guaranteed on those non-Euclidean spaces and, in fact, the system dynamics often possess multiple attractors (see, for example, DeVille [23] and reference therein). This is in contrast to the standard consensus dynamics over Euclidean spaces either in 


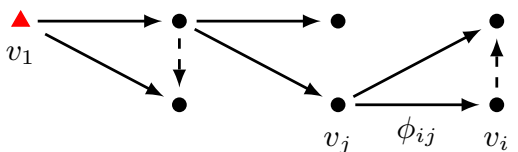

Fig. 1: Rooted digraph $G$. Nodes represent agents, and relative attitude information $\phi_{i j}$ is available if and only if $v_{j} v_{i}$ belongs to $E$. The red triangular node represents the (fully actuated) leader. A directed spanning tree $T$ of $G$ can be obtained by removing the dashed edges, which is realized by setting the corresponding controls $u_{i j}$ equal to zero for all time.

discrete- or continuous-time [24]-[29]. Thus, in these cases, controlling the agents by manipulating the tuning parameters becomes essential for escaping from undesired, but stable, configurations.

We let $\mathcal{U} \subseteq \mathbb{R}^{3 \times 3}$ be the collection of tuning parameters, which can be viewed as the set of admissible control inputs for the steering problem. The larger the set $\mathcal{U}$ is, the easier it is for the controller to steer the system. On the other hand, having a larger $\mathcal{U}$ implies that more information needs to be sent by the controller in real-time communication. In this paper, we focus on a relatively small set $\mathcal{U}$ that comprises only the scalar multiples of the identity, i.e.,

$$
\mathcal{U}:=\{u I \mid u \in \mathbb{R}\} .
$$

We investigate the feasibility of steering the agents using tuning parameters limited to the above set $\mathcal{U}$.

We now introduce the system model and controllability problem precisely. We assume that the leader $v_{1}$ is fully actuated. Specifically, let $e_{1}, e_{2}$, and $e_{3}$ be the standard basis of $\mathbb{R}^{3}$. We let

$$
\Omega_{a}:=e_{b} e_{c}^{\top}-e_{c} e_{b}^{\top},
$$

where $(a, b, c)$ is a cyclic rotation of $(1,2,3)$. Then, the dynamics of the leader are given by

$$
\dot{\theta}_{1}(t)=\sum_{a=1}^{3} u_{a}(t) \theta_{1}(t) \Omega_{a}
$$

where each $u_{a}(t)$ is a scalar control input. For the followers, the system dynamics are adapted from (5) and (6):

$$
\dot{\theta}_{i}(t)=\sum_{v_{j} \in V_{i}^{+}} u_{i j}(t) \theta_{i}(t)\left(\phi_{i j}(t)-\phi_{j i}(t)\right),
$$

where each $u_{i j}(t)$ is a scalar control input. Combining (8) and (9), we arrive at the following dynamics for the entire formation system:

$$
\dot{\theta}_{i}= \begin{cases}\sum_{a=1}^{3} u_{a} \theta_{i} \Omega_{a}, & \text { if } v_{i}=v_{1}, \\ \sum_{v_{j} \in V_{i}}^{+} u_{i j} \theta_{i}\left(\phi_{i j}-\phi_{j i}\right), & \text { otherwise. }\end{cases}
$$

The time arguments have been omitted in the above equation for ease of presentation. This paper investigates the relation between controllability of (10) and the structure of $G$. A main contribution of this work is to show that the formation system (10) is controllable as long as $G$ is a rooted directed graph with $v_{1}$ a root (see Fig. 1).

The remainder of the paper is organized as follows. We introduce preliminaries and state the main result in Section II. An outline of the proof of the main result will be given after the statement. A complete proof of the main result, along with detailed analysis, is given in Section III. We also propose a method of steering between target configurations in that Section. The paper ends with conclusions.

\section{Preliminaries and Main Result}

We now present basic notions and preliminaries on graph theory (Section II-A), gradient flows on the special orthogonal group (Section II-B), and theory of control-linear systems (Section II-C) before stating the main result of the paper, on the controllability of the formation system (10), in Section II-D. An outline of the proof will be given after the statement.

\section{A. Basic Notions from Graph Theory}

Let $G=(V, E)$ be a directed graph (or simply digraph), with $V=\left\{v_{1}, \ldots, v_{n}\right\}$ the node set and $E$ the edge set. An edge from $v_{i}$ to $v_{j}$ is denoted by $v_{i} v_{j} \in E$. The node $v_{j}$ is said to be an out-neighbor of $v_{i}$ and, correspondingly, $v_{i}$ is an in-neighbor of $v_{j}$. We denote by $V_{i}^{-}$and $V_{i}^{+}$the set of out-neighbors and in-neighbors of $v_{i}$, respectively.

A path from $v_{i}$ to $v_{j}$ is a sequence of nodes $v_{p_{1}}, \ldots, v_{p_{n}}$, with $v_{p_{1}}=v_{i}$ and $v_{p_{n}}=v_{j}$, such that each $v_{p_{k}} v_{p_{k+1}}$, for $k=1, \ldots, n-1$, is an edge of $G$ and there is no repetition of nodes in the sequence. The length of the path is the number of edges contained in it.

A subgraph $G^{\prime}=\left(V^{\prime}, E^{\prime}\right)$ of $G$ satisfies $V^{\prime} \subseteq V$ and $E^{\prime} \subseteq E$. Given a subset $V^{\prime}$ of $V$, a subgraph $G^{\prime}=\left(V^{\prime}, E^{\prime}\right)$ is said to be induced by $V^{\prime}$ if the edge set $E^{\prime}$ satisfies the following condition: for any two nodes $v_{i}$ and $v_{j}$ in $V^{\prime}, v_{i} v_{j}$ is an edge of $G^{\prime}$ if and only if it is an edge of $G$.

A digraph $G$ is rooted if there is a node $v_{1}$ such that for any other $v_{i}$ in $G$, there is a path from $v_{1}$ to $v_{i}$. The node $v_{1}$ is a root of $G$. A digraph $G$ is acyclic if it does not contain any cycle as its subgraph. If $G$ is also rooted, then it has a unique root $v_{1}$. A directed tree $T$ is a special rooted, acyclic digraph such that every node, except the root node $v_{1}$, has only one in-neighbor. A node $v_{i}$ is a leaf of the directed tree if it does not have any out-neighbors $\left(V_{i}^{-}=\varnothing\right)$. For any given node $v_{i}$ other than $v_{1}$, there is a unique path from $v_{1}$ to $v_{i}$. The depth of the node $v_{i}$ is the length of that path. The depth of the root $v_{1}$ is 0 by default. The depth of the tree is the maximal value of depths of all the nodes. Note that if $G=(V, E)$ is rooted with $v_{1}$ a root, then it contains as a subgraph a directed tree $T=\left(V, E^{\prime}\right)$, with the same node set $V$, such that $v_{1}$ is the root of $T$. The subgraph $T$ is called a directed spanning tree of $G$ (see Fig. 1).

\section{B. Normal Metric and Gradient Flow}

In this section, we show that (5) is the gradient flow of (4) with respect to the normal metric. To that end, we let $T_{\theta} \mathrm{SO}(3)$ be the tangent space of $\mathrm{SO}(3)$ at $\theta$. Let $\mathfrak{s o}(3)$ be the space of all $3 \times 3$ skew-symmetric matrices. Then,

$$
T_{\theta} \mathrm{SO}(3)=\{\theta \Omega \mid \Omega \in \mathfrak{s o}(3)\} .
$$

The so-called normal metric $\langle\cdot, \cdot\rangle$ on $\mathrm{SO}(3)$ is

$$
\left\langle\theta \Omega_{1}, \theta \Omega_{2}\right\rangle_{\theta}:=-\operatorname{tr}\left(\Omega_{1} \Omega_{2}\right), \quad \forall \theta \in \operatorname{SO}(3) .
$$


Let $\xi: \mathrm{SO}(3) \rightarrow \mathbb{R}$ be an arbitrary continuously differentiable function. For a vector $W \in T_{\theta} \mathrm{SO}(3)$, we denote by $D_{W} \xi(\theta)$ the directional derivative of $\xi$ at $\theta$ along $W$ :

$$
D_{W} \xi(\theta):=\lim _{\epsilon \rightarrow 0} \frac{\xi(\theta+\epsilon W)-\xi(\theta)}{\epsilon} .
$$

Then, the gradient of $\xi$ at $\theta, \nabla \xi(\theta)$, with respect to the normal metric is a vector in $T_{\theta} \mathrm{SO}(3)$ that satisfies

$$
\langle\nabla \xi(\theta), W\rangle_{\theta}=D_{W} \xi(\theta), \quad \forall W \in T_{\theta} \mathrm{SO}(3) .
$$

Because the metric $\langle\cdot, \cdot\rangle_{\theta}$ is positive definite, the above condition determines $\nabla \xi(\theta)$ uniquely.

In the case here, the potential function $\xi_{i}$ for agent $v_{i}$ is given by

$$
\xi_{i}\left(\theta_{i}\right)=\sum_{v_{j} \in V_{i}^{+}}\left\|\theta_{i}^{\top} \theta_{j}-U_{i j}\right\|_{F}^{2},
$$

where only $\theta_{i}$ is treated as a variable (so that the gradient of $\xi_{i}\left(\theta_{i}\right)$ may be considered as the partial derivative of $\xi_{i}\left(\theta_{1}, \ldots, \theta_{N}\right)$ with respect to $\left.\theta_{i}\right)$. For any tangent vector $W=\theta_{i} \Omega$, we obtain by computation

$$
D_{W} \xi_{i}\left(\theta_{i}\right)=\operatorname{tr}\left(\Omega \sum_{v_{j} \in V_{i}^{+}}\left(\phi_{i j} U_{i j}^{\top}-U_{i j} \phi_{j i}\right)\right) .
$$

To find the potential function gradient $\nabla \xi_{i}\left(\theta_{i}\right) \in T_{\theta_{i}} \mathrm{SO}(3)$, we set $\nabla \xi_{i}\left(\theta_{i}\right)=\theta_{i} \Omega^{*}$ for some $\Omega^{*} \in \mathfrak{s o}(3)$, where $\Omega^{*}$ is to be determined (and depends on $\theta_{i}$ and the $\theta_{j}$ ). Using the defining condition (11), we have

$-\operatorname{tr}\left(\Omega^{*} \Omega\right)=\operatorname{tr}\left(\Omega \sum_{v_{j} \in V_{i}^{+}}\left(\phi_{i j} U_{i j}^{\top}-U_{i j} \phi_{j i}\right)\right), \forall \Omega \in \mathfrak{s o}(3)$.

Note that the matrix on the right-hand side of the above expression is skew-symmetric since $\phi_{j i}=\phi_{i j}^{\top}$. It thus follows that

$$
\Omega^{*}=-\sum_{v_{j} \in V_{i}^{+}}\left(\phi_{i j} U_{i j}^{\top}-U_{i j} \phi_{j i}\right),
$$

resulting in the gradient dynamics (5). In particular, if $U_{i j}=u_{i j} I$ is a scalar multiple of identity, then

$$
\Omega^{*}=-\sum_{v_{j} \in V_{i}^{+}} u_{i j}\left(\phi_{i j}-\phi_{j i}\right) .
$$

\section{Control-linear Systems}

Consider the following control system on a differentiable manifold $M$ embedded in an Euclidean space:

$$
\dot{x}(t)=\sum_{i=1}^{m} u_{i}(t) f_{i}(x(t)),
$$

where each $u_{i}(t)$ is a scalar control input and each $f_{i}$ is a control vector field. We first have the following definition about controllability of (12) over an arbitrary subset of $M$ :

Definition 1. Let $M^{\prime}$ be an open and path-connected subset of $M$. System (12) is controllable over $\boldsymbol{M}^{\prime}$ if for any initial condition $x(0) \in M^{\prime}$, any target $\hat{x} \in M^{\prime}$, and any time $\tau>0$, there are bounded, integrable functions $u_{i}:[0, \tau] \rightarrow \mathbb{R}$, for $i=1, \ldots, m$, as control inputs such that the solution $x(t)$ generated by (12) belongs to $M^{\prime}$ for all $t \in[0, \tau]$ and, moreover, $x(\tau)=\hat{x}$. If $M^{\prime}$ can be chosen to be the entire space $M$, then system (12) is controllable.
The path-connectivity of the subset $M^{\prime}$ is necessary as the solution $x(t)$ is a continuous path that connects the two points $x(0)$ and $\hat{x}$ in $M^{\prime}$. The openness of $M^{\prime}$ is not required, but is often needed for local controllability analysis [30]. If, further, $M^{\prime}$ is dense in $M$, then system (12) is controllable almost everywhere. It is well known that system (12) is controllable over $M^{\prime}$ if it satisfies the so-called Lie algebra rank condition (LARC) over the subset. We review this fact, also known as the Chow-Rashevsky theorem [31], below.

First, for any two given vector fields $f_{1}$ and $f_{2}$ on $M$, we let $\left[f_{1}, f_{2}\right]$ be the Lie bracket, which produces another vector field on $M$ defined as follows:

$$
\left[f_{1}, f_{2}\right](x):=\frac{\partial f_{2}}{\partial x}\left(f_{1}(x)\right)-\frac{\partial f_{1}}{\partial x}\left(f_{2}(x)\right), \quad \forall x \in M .
$$

We now let $L$ be the finite-dimensional vector space (over $\mathbb{R}$ ) spanned by $\left\{f_{1}, \ldots, f_{m}\right\}$ and $\mathcal{L}$ be the Lie algebra generated by $L$, i.e., the smallest vector space that contains $L$ as a subspace and is closed under the Lie bracket.

For a given point $x \in M$, we let $\mathcal{L}_{x}$ be the evaluation of the Lie algebra $\mathcal{L}$ at $x$ :

$$
\mathcal{L}_{x}:=\{h(x) \mid h \in \mathcal{L}\} .
$$

Note that each $\mathcal{L}_{x}$ is a subspace of the tangent space of $M$ at $x$, which we denote by $T_{x} M$. We now recall the $L A R C$ :

Lemma 1 ([31]). Let $M^{\prime}$ be an open and path-connected subset of $M$. If $\mathcal{L}_{x}=T_{x} M$ for all $x \in M^{\prime}$, then system (12) is controllable over $M^{\prime}$.

We next introduce the notion of $k$ th bracket controllability. To that end, we define induction vector spaces $\mathcal{L}(k)$, for $k \geq 0$, as follows: For $k=0$, we let $\mathcal{L}(0):=L$ and for $k \geq 1$, we let

$$
\mathcal{L}(k):=[\mathcal{L}(k-1), L],
$$

which is, by convention, the vector space spanned by all the elements $\left[h_{1}, h_{2}\right]$ with $h_{1} \in \mathcal{L}(k-1)$ and $h_{2} \in L$. We call an element $h \in \mathcal{L}$ a Lie product if $h$ belongs to $\mathcal{L}(k)$ for some $k \geq 0$. The depth of the Lie product $h$ is the number of Lie brackets required to produce it: if $h \in \mathcal{L}(k)$, then the depth of $h$ is $k$. Note that every element $h$ in $\mathcal{L}$ can be written as a linear combination $h=\sum_{i=0}^{n} h_{i}$, with each $h_{i}$ belonging to $\mathcal{L}\left(k_{i}\right)$ for some $k_{i} \geq 0$. This holds due to the anti-symmetry of Lie brackets and the Jacobi identity. For example, we can write the iterative Lie bracket $\left[\left[f_{1}, f_{2}\right],\left[f_{3}, f_{4}\right]\right] \in \mathcal{L}$ as

$$
\left[\left[f_{1}, f_{2}\right],\left[f_{3}, f_{4}\right]\right]=\left[\left[\left[f_{1}, f_{2}\right], f_{3}\right], f_{4}\right]-\left[\left[\left[f_{1}, f_{2}\right], f_{4}\right], f_{3}\right],
$$

where each of the right-hand side terms are in $\mathcal{L}(3)$ (but the left-hand side is not in any $\mathcal{L}(k)$ ).

For convenience, we further let

$$
\mathcal{L}[k]:=\sum_{\ell=0}^{k} \mathcal{L}(\ell),
$$

defined as the vector space spanned by all the elements in $\mathcal{L}(\ell)$ for all $\ell \leq k$. Note that we have the following increasing sequence:

$$
L:=\mathcal{L}[0] \subseteq \mathcal{L}[1] \subseteq \mathcal{L}[2] \subseteq \cdots \subseteq \mathcal{L}[\infty]=\mathcal{L} .
$$


Similarly, we let

$$
\mathcal{L}_{x}[k]:=\{h(x) \mid h \in \mathcal{L}[k]\}
$$

be the evaluation of $\mathcal{L}[k]$ at $x$. We now have the following definition for $k$ th bracket controllability:

Definition 2. Let $M^{\prime}$ be an open and path-connected subset of M. System (12) is kth bracket controllable over $\boldsymbol{M}^{\prime}$, for $k \geq 0$, if $\mathcal{L}_{x}[k]=T_{x} M$ for all $x \in M^{\prime}$.

From (13), if system (12) is $k$ th bracket controllable over $M^{\prime}$ for some $k$, then it is $\ell$ th bracket controllable over $M^{\prime}$ for all $\ell \geq k$. We also note that if $M^{\prime}$ and $M^{\prime \prime}$ are two open and path-connected subsets of $M$, with $M^{\prime \prime} \subseteq M^{\prime}$, and if system (12) is $k$ th bracket controllable over $M^{\prime}$, then by Def. 2 the system is also $k$ th bracket controllable over the smaller subset $M^{\prime \prime}$. In general, the fewer Lie brackets required, the easier it is for the controller to generate inputs to steer the system within $M^{\prime}$. A small number $k$ is thus desired for motion planning.

\section{Main Result}

In this section, we state the main results about controllability of the formation system (10). Recall that agent $v_{1}$ is the leading agent whose dynamics is fully actuated and all the other agents are followers whose dynamics are adapted from the gradient flows (5). For convenience, we reproduce below the (control-linear) system dynamics:

$$
\dot{\theta}_{i}= \begin{cases}\sum_{a=1}^{3} u_{a} \theta_{i} \Omega_{a}, & \text { if } v_{i}=v_{1}, \\ \sum_{v_{j} \in V_{i}}^{+} u_{i j} \theta_{i}\left(\phi_{i j}-\phi_{j i}\right), & \text { otherwise }\end{cases}
$$

where $\Omega_{a}$ and $\phi_{i j}$ are defined in (7) and (3), respectively. Let

$$
\Theta(t):=\left[\theta_{1}(t) ; \cdots ; \theta_{N}(t)\right]
$$

be the collection of the states of the $N$ individual agents at time $t$. We call $\Theta(t)$ a configuration of the formation system. Correspondingly, let

$$
P:=\operatorname{SO}(3)^{N}
$$

be the configuration space. In case we need to emphasize the underlying network topology $G$, we will write $P(G)$. During the analysis, we will consider different subformation systems of (14) induced by subgraphs of $G$ : a subformation system induced by $G^{\prime}=\left(V^{\prime}, E^{\prime}\right)$ is obtained by considering only the agents $v_{i} \in V^{\prime}$ and setting $u_{i j}=0$ for all time if $v_{j} v_{i}$ does not belong to $E^{\prime}$. Correspondingly, we will use $P\left(G^{\prime}\right)$ to denote the configuration space associated with the subformation system.

We are now in a position to state the main result of the paper.

Theorem 1. Let $G$ be a rooted digraph with $v_{1}$ the root. Then, system (14) is controllable over the entire configuration space $P$.

We provide below an outline of the proof of Theorem 1 . Detailed arguments will be given in the next section.

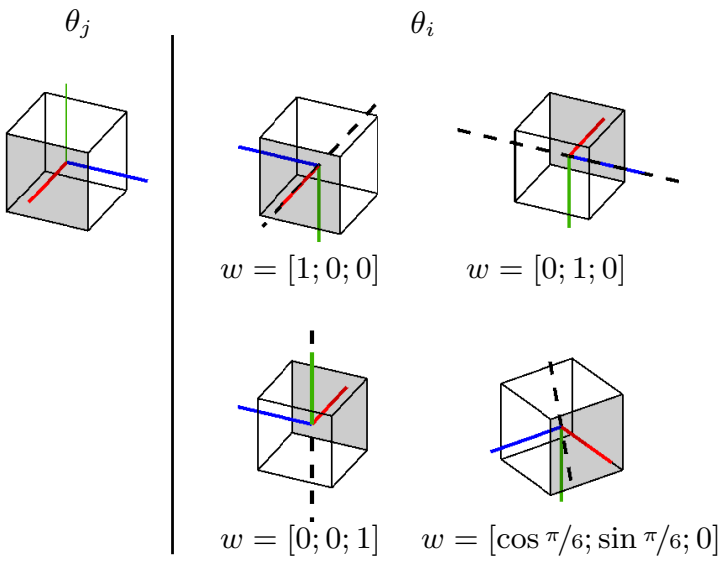

Fig. 2: Illustration of relative attitudes $\phi_{i j}=\theta_{i}^{\top} \theta_{j}$ between agents $v_{i}$ and $v_{j}$. The attitude of each agent is represented by an orthonormal frame (with the three axes highlighted in red, blue, and green, respectively). The attitude $\theta_{j}$ of agent $v_{j}$ is shown on the left and is fixed. Four different attitudes $\theta_{i}$ of agent $v_{i}$ are shown on the right. Every such $\theta_{i}$ can be obtained by a rotation of $\pi$ of $\theta_{j}$ about a certain axis. The axis is represented by the dashed line and is aligned with a vector $w$. Consequently, for every attitude $\theta_{i}$ shown in the figure, the relative attitude $\phi_{i j}$ belongs to $\Phi$.

Outline of proof. The controllability analysis revolves around a key subset of the configuration space $P$, termed the set of nonsingular configurations. We introduce the set below.

To proceed, we first introduce a subset of $\mathrm{SO}(3)$ defined as follows:

$$
\Phi:=\left\{-I+2 w w^{\top} \mid w \in \mathbb{R}^{3} \text { with }\|w\|=1\right\} .
$$

Algebraically, the set $\Phi$ comprises all symmetric special orthogonal matrices in $\mathrm{SO}(3)$ whose eigenvalues are given by $-1,-1$, and 1 ; the only other symmetric, special orthogonal matrix is the identity matrix. Note that $w$ is the eigenvector of $\left(-I+2 w w^{\top}\right)$ corresponding to the eigenvalue 1 . Geometrically, $\Phi$ comprises all special orthogonal matrices representing a rotation of $\pi$ of a rigid body around a certain axis (which is aligned with the vector $w$ ) that runs through a fixed point of the rigid body. In Fig. 2, we illustrate pairs of rigid bodies with relative attitudes belonging to the set $\Phi$.

Since the underlying digraph $G$ of the formation system (14) is rooted, it contains a directed spanning tree $T$ with $v_{1}$ the root. Note that $G$ may have several different directed spanning trees; we choose one and fix it in the sequel.

For each edge $v_{j} v_{i}$ of $T$ and for each configuration $\Theta=\left[\theta_{1} ; \cdots ; \theta_{N}\right]$, we recall that $\phi_{i j}=\theta_{i}^{\top} \theta_{j}$ is the relative attitude between agents $v_{i}$ and $v_{j}$. For the given edge, we let $P_{i j}$ be the collection of configurations with $\phi_{i j}$ belonging to $\Phi$, i.e.

$$
P_{i j}:=\left\{\Theta \in P \mid \phi_{i j}=\theta_{i}^{\top} \theta_{j} \in \Phi\right\} .
$$

Since every matrix in $\Phi$ is symmetric, if $\phi_{i j} \in \Phi$, then $\phi_{j i} \in \Phi$. We further define

$$
P_{\mathrm{s}}:=\cup_{v_{j} v_{i} \in E^{\prime}} P_{i j} \quad \text { and } \quad P_{\mathrm{ns}}:=P \backslash P_{\mathrm{s}} .
$$

Note that the definitions of $P_{\mathrm{s}}$ and $P_{\mathrm{ns}}$ implicitly depend on the choice of spanning tree $T$. If we need to indicate explicitly the dependence, we will write $P_{\mathrm{s}}(T)$ and $P_{\mathrm{ns}}(T)$. The two sets 
defined in (17) are particularly relevant to us in determining the depth of Lie products required to make the $L A R C$ hold. We introduce the following definition:

Definition 3. If a configuration $\Theta$ belongs to $P_{\mathrm{S}}(T)$, then it is singular with respect to $T$. Otherwise, $\Theta$ is nonsingular.

The "singularity" of a configuration $\Theta \in P_{\mathrm{s}}$ comes from the fact that the computational results for Lie brackets of control vector fields of the formation system (14) differ from those for the case where $\Theta \in P_{\mathrm{ns}}$. We will elaborate on this in the next section. With the above definition of (non)singular configurations, we provide below the major components of the proof of Theorem 1:

1) Topological properties of $P_{\mathrm{ns}}$. We show that the set of nonsingular configurations $P_{\mathrm{ns}}$ is open and dense in $P$ by identifying each $P_{i j}$, defined in (16), with a submanifold of $P$ of codimension 1 . It then follows that $P_{\mathrm{ns}}$ can be realized by removing from $P$ a finite union of submanifolds of codimension 1 , which implies that $P_{\mathrm{ns}}$ is open and dense. Besides openness and density of $P_{\mathrm{ns}}$, we further show that the set $P_{\mathrm{ns}}$ is path-connected. The proof will be carried out by explicit construction of a continuous path that connects two arbitrary configurations in $P_{\mathrm{ns}}$. These topological properties of $P_{\mathrm{ns}}$ will be established in Section III-A.

2) Controllability over $P_{\mathrm{ns}}$. We next show that the formation system (14) is $d$ th bracket controllable over the set of nonsingular configurations $P_{\mathrm{ns}}$, where $d$ is the depth of the directed spanning tree $T$ of $G$. The proof of this result relies on the use of the $L A R C$ (see Lemma 1). By explicit computation, we show that the $L A R C$ can be satisfied over any nonsingular configuration if all the Lie products of the control vector fields of depth less than or equal to $d$ are included. Note that the result holds under the assumption that the configurations are nonsingular. The computation of the Lie brackets will be carried out in Section III-B.

3) Complete controllability. Finally, we bridge the gap between "controllability almost everywhere" (since $P_{\mathrm{ns}}$ is open, dense, and path connected) and "controllability everywhere." Specifically, we show that it is feasible to steer from every singular (resp. nonsingular) configuration to every nonsingular (resp. singular) configuration. This will be established by induction on the number of agents. The proof is given in Section III-C.

\section{ANAlysis AND PRoOf of TheOrem 1}

We will now follow the steps outlined at the end of the last section to establish Theorem 1.

\section{A. Topological Properties of Nonsingular Configurations}

Let $G=(V, E)$ be a rooted digraph and $T=\left(V, E^{\prime}\right)$ be a directed spanning tree of $G$. We will establish the following result:

Proposition 2. The set of nonsingular configurations $P_{\mathrm{ns}}(T)$ is open, dense in $P$ and, moreover, path-connected.
The proposition will be established after a sequence of lemmas. To proceed, we first recall that the set $\Phi$ is a subset of $\mathrm{SO}(3)$ defined in (15). Each matrix $\phi \in \Phi$ is symmetric and has eigenvalues $-1,-1$, and 1 . Let $\mathbb{R P}^{2}$ be the real projective space defined as the manifold of all straight lines in $\mathbb{R}^{3}$ passing through the origin. We make the following observation:

Lemma 2. The set $\Phi$ is diffeomorphic to $\mathbb{R P}^{2}$.

Proof. Let $w_{1}$ and $w_{2}$ be two vectors in $\mathbb{R}^{3}$ of unit norm. Suppose that two matrices $\left(-I+w_{1} w_{1}^{\top}\right)$ and $\left(-I+w_{2} w_{2}^{\top}\right)$ in $\Phi$ are identical to each other; then, we have that $w_{1}^{\top} w_{1}=w_{2}^{\top} w_{2}$, which holds if and only if $w_{1}=w_{2}$ or $w_{1}=-w_{2}$. We thus conclude that $\Phi \approx \mathbb{R P}^{2}$.

We next recall that the set of singular configurations $P_{\mathrm{s}}$ is defined as the union of $P_{i j}$, for $v_{j} v_{i} \in E^{\prime}$, where each $P_{i j}$ is defined in (16) by requiring that the relative attitude $\phi_{i j}$ belongs to the set $\Phi$. We now have the following fact:

Lemma 3. Each $P_{i j}$ is diffeomorphic to $\mathrm{SO}(3)^{N-1} \times \mathbb{R P}^{2}$.

Proof. Note that by (16), each $P_{i j}$ is diffeomorphic to $\operatorname{SO}(3)^{N-1} \times \Phi$. To see this, we let $\Theta=\left[\theta_{1} ; \cdots ; \theta_{N}\right]$ be a configuration in $P$ and $\Theta_{-j}^{\prime} \in \mathrm{SO}(3)^{N-1}$ be obtained from $\Theta$ by omitting its $j$ th component $\theta_{j}$. Then, the map

$$
\rho: P_{i j} \rightarrow \mathrm{SO}(3)^{N-1} \times \Phi
$$

defined by

$$
\rho: \Theta \mapsto\left(\Theta_{-j}^{\prime}, \theta_{i}^{\top} \theta_{j}\right)
$$

is a diffeomorphism. The inverse $\rho^{-1}$ sends a pair $\left(\Theta_{-j}^{\prime}, \phi\right) \in \mathrm{SO}(3)^{N-1} \times \Phi$ to a singular configuration $\Theta \in P_{\mathrm{s}}$ obtained by inserting $\theta_{i} \phi$ into $\Theta_{-j}^{\prime}$ so that $\theta_{i} \phi$ is the $j$ th component of $\Theta$. The lemma then follows from the fact that $\Phi$ is diffeomorphic to $\mathbb{R P}^{2}$.

To establish Prop. 2, we further need the following fact:

Lemma 4. For any $\phi \in \mathrm{SO}(3)$, there exists a $\psi \in \mathrm{SO}(3)$ and an angle $\alpha \in(-\pi, \pi]$ such that

$$
\psi \phi \psi^{\top}=R_{\alpha}:=\left[\begin{array}{ccc}
1 & 0 & 0 \\
0 & \cos \alpha & -\sin \alpha \\
0 & \sin \alpha & \cos \alpha
\end{array}\right] .
$$

In particular, the matrix $\phi$ belongs to $\Phi$ if and only if $\alpha=\pi$.

Proof. The decomposition (18) directly follows from Euler's rotation theorem [32]. Since $\psi \phi \psi^{\top}$ is a similarity transformation, from (18), the eigenvalues of $\phi$ are $e^{\mathrm{i} \alpha}, e^{-\mathrm{i} \alpha}$, and 1 . Thus, $\phi$ belongs to $\Phi$ if and only if $\alpha=\pi$.

With the above preliminaries, we now prove Prop. 2:

Proof of Prop. 2. We first show that $P_{\mathrm{ns}}$ is open and dense in $P$. By Lemma 3, the codimension of each $P_{i j}$ is 1 , i.e., $\operatorname{dim}(P)-\operatorname{dim}\left(P_{i j}\right)=1$. Because $P_{\mathrm{ns}}=P \backslash P_{\mathrm{s}}$ and because $P_{\mathrm{s}}$ is a finite union of $P_{i j}$, we have that $P_{\mathrm{ns}}$ is open and dense in $P$.

We now show that $P_{\mathrm{ns}}$ is path-connected. The proof will be carried out by induction on $N$, the number of nodes in $G$. For the base case, $N=1$, there does not exist any singular configuration, so $P_{\mathrm{ns}}=P=\mathrm{SO}(3)$. The result then follows 
from the fact that $\mathrm{SO}(3)$ is path-connected. For the inductive step, we assume that the result holds for $(N-1)$ and prove for $N$

Consider two nonsingular configurations

$$
\Theta=\left[\theta_{1} ; \cdots ; \theta_{N}\right] \text { and } \hat{\Theta}=\left[\hat{\theta}_{1} ; \cdots ; \hat{\theta}_{N}\right] .
$$

We exhibit below a path $p$ in $P_{\text {ns }}$ that connects $\Theta$ and $\hat{\Theta}$. Note that we are now using the term 'path' to describe a continuous function from a closed interval to $P$, as opposed to a sequence of nodes in $G$. The path $p$ will be obtained by concatenating three subpaths $p_{1}, p_{2}$, and $p_{3}$, which will be constructed subsequently.

We first construct $p_{1}$. Without loss of generality, we assume that $v_{N}$ is a leaf of the directed spanning tree $T$ and $v_{N-1}$ is the in-neighbor of $v_{N}$ within $T$. Let $\phi_{N-1, N}:=\theta_{N-1}^{\top} \theta_{N}$ be the relative attitude. Using Euler's rotation theorem, we let $\phi_{N-1, N}=\psi^{\top} R_{\alpha} \psi$, where $R_{\alpha}$ is the matrix defined in (18), with $\alpha \in(-\pi, \pi]$. Because $\Theta$ is nonsingular, $\phi_{N-1, N}$ does not belong to $\Phi$. It follows from Lemma 4 that $\alpha \neq \pi$. We then define the subpath $p_{1}:[0,1] \rightarrow P$ as follows:

$$
p_{1}(t):=\left[\theta_{1} ; \cdots ; \theta_{N-1} ; \theta_{N-1} \psi^{\top} R_{(1-t) \alpha} \psi\right] .
$$

Along the path $p_{1}$, only the relative attitude between agents $v_{N}$ and $v_{N-1}$, which is given by $\psi^{\top} R_{(1-t) \alpha} \psi$, changes. Since $\alpha<\pi,(1-t) \alpha \neq \pi$ for all $t \in[0,1]$. By Lemma 4, the relative attitude $\psi^{\top} R_{(1-t) \alpha} \psi$ does not belong to $\Phi$, so $p_{1}(t)$ lies in $P_{\mathrm{ns}}$ for all $t \in[0,1]$. By the construction of $p_{1}$,

$$
p_{1}(0)=\Theta \quad \text { and } \quad p_{1}(1)=\left[\theta_{1} ; \ldots, \theta_{N-1} ; \theta_{N-1}\right]
$$

since $R_{0}=I$.

We next construct $p_{2}$. Let $G^{\prime}$ and $T^{\prime}$ be the subgraphs of $G$ and $T$ induced by the first $(N-1)$ nodes. Since $v_{N}$ is a leaf of $T, T^{\prime}$ is a directed spanning tree of $G^{\prime}$. Consider the following subconfigurations of $\Theta$ and $\hat{\Theta}$ :

$$
\Theta^{\prime}:=\left[\theta_{1} ; \cdots ; \theta_{N-1}\right] \text { and } \hat{\Theta}^{\prime}:=\left[\hat{\theta}_{1} ; \cdots ; \hat{\theta}_{N-1}\right] .
$$

Because $\Theta$ and $\hat{\Theta}$ belong to $P_{\mathrm{ns}}(T)$ and because $T^{\prime}$ is a subgraph of $T$, we have that both $\Theta^{\prime}$ and $\hat{\Theta}^{\prime}$ belong to $P_{\mathrm{ns}}\left(T^{\prime}\right)$. Thus, by the induction hypothesis, there exists a continuous path $q:[0,1] \rightarrow P_{\mathrm{ns}}\left(T^{\prime}\right)$ such that $q(0)=\Theta^{\prime}$ and $q(1)=\hat{\Theta}^{\prime}$. Let $q_{N-1}(t)$ be the last component of $q(t)$. We then define the subpath $p_{2}:[0,1] \rightarrow P$ by augmenting $q$ as

$$
p_{2}(t):=\left[q(t) ; q_{N-1}(t)\right] \text {. }
$$

By construction, the attitudes of agents $v_{N-1}$ and $v_{N}$ are identical to each other along the path $p_{2}$. In particular, the relative attitude between the two agents is the identity matrix, which does not belong to the set $\Phi$. Combining this with the fact that $q(t) \in P_{\mathrm{ns}}\left(T^{\prime}\right)$ for all $t \in[0,1]$, we have that the entire subpath $p_{2}$ is in $P_{\mathrm{ns}}(T)$. Note that

$$
p_{2}(0)=p_{1}(1) \quad \text { and } \quad p_{2}(1)=\left[\hat{\theta}_{1}, \ldots, \hat{\theta}_{N-1}, \hat{\theta}_{N-1}\right] .
$$

Finally, the construction of the subpath $p_{3}$ is similar to that of $p_{1}$. We let $\hat{\phi}_{N-1, N}:=\hat{\theta}_{N-1}^{\top} \hat{\theta}_{N}$ and decompose it as $\hat{\phi}_{N-1, N}=\hat{\psi}^{\top} R_{\hat{\alpha}} \hat{\psi}$. Since $\hat{\Theta}$ is nonsingular, $\hat{\alpha} \neq \pi$. We then define

$$
p_{3}(t):=\left[\hat{\theta}_{1} ; \cdots ; \hat{\theta}_{N-1} ; \hat{\theta}_{N-1} \hat{\psi}^{\top} R_{t \hat{\alpha}} \hat{\psi}\right] .
$$

Since $\hat{\alpha}<\pi$, we have that $t \hat{\alpha} \neq \pi$ for all $t \in[0,1]$. Thus, the entire path $p_{3}$ is in $P_{\mathrm{ns}}$. Note that

$$
p_{3}(0)=p_{2}(1) \quad \text { and } \quad p_{3}(1)=\hat{\Theta} .
$$

By concatenating $p_{1}, p_{2}$, and $p_{3}$, we obtain a continuous path $p$ in $P_{\mathrm{ns}}$ that connects $\Theta$ and $\hat{\Theta}$.

\section{B. Controllability over Nonsingular Configurations}

In this section, we establish the following result:

Proposition 3. Let $G$ be rooted and $T$ be a directed spanning tree of $G$, with $v_{1}$ the root and $d$ the depth. Then, system (14) is dth bracket controllable over $P_{\mathrm{ns}}(T)$.

The proof of the result will rely on the computation of Lie brackets of control vector fields over the set $P_{\mathrm{ns}}$. To proceed, we first note that the tangent space of $P$ at $\Theta=\left[\theta_{1} ; \cdots ; \theta_{N}\right]$ is given by the Cartesian product

$$
T_{\Theta} P=T_{\theta_{1}} \mathrm{SO}(3) \times \cdots \times T_{\theta_{N}} \mathrm{SO}(3) .
$$

For a given tangent vector

$$
\left[\theta_{1} A_{1} ; \cdots ; \theta_{N} A_{N}\right] \in T_{\Theta} P,
$$

with $A_{i} \in \mathfrak{s o}(3)$ for all $i=1, \ldots, N$, we refer to $\theta_{k} A_{k}$ as the $k$ th component of the vector.

We next introduce shorthand notations for the control vector fields. For each $a=1,2,3$, we let

$$
f_{a}(\Theta):=\left[\theta_{1} \Omega_{a} ; 0 ; \cdots ; 0\right],
$$

where $\Omega_{a}$ is defined in (7). For each edge $v_{j} v_{i}$ of $G$, we define the vector field $g_{i j}$ by specifying its $k$ th component as

$$
\begin{aligned}
& \text { the } k \text { th component of } g_{i j}(\Theta):= \\
& \qquad \begin{cases}\theta_{i}\left(\phi_{i j}-\phi_{j i}\right), & \text { if } v_{k}=v_{i}, \\
0, & \text { otherwise, }\end{cases}
\end{aligned}
$$

where $\phi_{i j}=\theta_{i}^{\top} \theta_{j}$ as defined in (3). With the above notations, we re-write the formation system (14) as

$$
\dot{\Theta}(t)=\sum_{a=1}^{3} u_{a}(t) f_{a}(\Theta(t))+\sum_{v_{j} v_{i} \in E} u_{i j}(t) g_{i j}(\Theta(t)) .
$$

We will now evaluate the Lie products of the control vector fields at a nonsingular configuration. The following computational result is key to the proof of Prop. 3:

Lemma 5. Let $v_{j} v_{i}$ be an edge of $G$ and $g_{i j}$ be defined in (20). Let $h$ be an arbitrary differentiable vector field on $P$ with $k$ th component $\theta_{k} A_{k}(\Theta)$ for some $A_{k}(\Theta) \in \mathfrak{s o}(3)$. Suppose that the ith component of $h$ is identically zero, and further, that no component of $h$ depends on $\theta_{i}$, i.e.,

$$
h=\left[\theta_{1} A_{1}\left(\Theta_{-i}^{\prime}\right) ; \cdots ; 0 ; \cdots ; \theta_{N} A_{N}\left(\Theta_{-i}^{\prime}\right)\right]
$$

where the zero is the ith component and $\Theta_{-i}^{\prime} \in \mathrm{SO}(3)^{N-1}$ is obtained by omitting $\theta_{i}$ from $\Theta$. Then, only the ith component of $\left[g_{i j}, h\right](\Theta)$ is nonzero and it is given by $\theta_{i}\left(\phi_{i j} A_{j}(\Theta)+A_{j}(\Theta) \phi_{j i}\right)$.

A proof of the result is provided in the Appendix. With the preliminaries above, we now establish Prop. 3. 
Proof of Prop. 3. The proof will be carried out by induction on the depth $d$ of the directed spanning tree $T$. For the base case where $d=0$, there is only one single node, namely $v_{1}$. The leader $v_{1}$ has dynamics (8) that are fully actuated, so the system is 0th bracket controllable everywhere. For the inductive step, we assume that the result holds for $(d-1)$ and prove for $d$. Specifically, we show that if $\Theta$ belongs to $P_{\mathrm{ns}}$, then $T_{\Theta} P=\mathcal{L}_{\Theta}[d]$.

Recall that the depth of a node $v_{i}$ within $T$ is the length of the unique path from the root $v_{1}$ to $v_{i}$. Let $V^{*}$ be the set of nodes in $T$ whose depths are $d$ and let $V^{\prime}:=V \backslash V^{*}$. Let $G^{\prime}$ and $T^{\prime}$ be the subgraphs of $G$ and $T$, respectively, induced by the subset $V^{\prime}$. Then, $T^{\prime}$ is a directed spanning tree of $G^{\prime}$ and the depth of $T^{\prime}$ is $(d-1)$. We further denote by $E^{\prime}$ the edge set of $G^{\prime}$.

Now, consider the subformation system formed by the agents $v_{j}$ in $V^{\prime}$. By the induction hypothesis, the subformation system is $(d-1)$ th bracket controllable over $P_{\mathrm{ns}}\left(T^{\prime}\right)$. More specifically, we let $\mathcal{L}^{\prime}$ be the Lie subalgebra of $\mathcal{L}$ generated by the collection of the $f_{a}$, for $a=1,2,3$, and the $g_{j \ell}$ for $v_{\ell} v_{j} \in E^{\prime}$. Note that if $h \in \mathcal{L}^{\prime}$, then it has zero as its $i$ th component, for any $v_{i} \in V^{*}$. This holds because it is constructed by taking Lie brackets of the $f_{a}$ and the $g_{j \ell}$, which, by (19) and (20), have zero as their $i$ th components. Further, since no agent $v_{j} \in V^{\prime}$ can have an agent $v_{i} \in V^{*}$ as its in-neighbor in $G^{\prime}, h$ does not depend on the $\theta_{i}$. For any $\Theta \in P(G)$, we let $\Theta^{\prime} \in P\left(G^{\prime}\right)$ be the corresponding subconfiguration formed by the $\theta_{j}$, for $v_{j} \in V^{\prime}$. Since $T^{\prime}$ is a subgraph of $T$, it follows that if $\Theta$ is in $P_{\mathrm{ns}}(T)$, then $\Theta^{\prime}$ is in $P_{\mathrm{ns}}\left(T^{\prime}\right)$. Thus, by the induction hypothesis, we have the following spanning condition for all $\Theta$ belonging to $P_{\mathrm{ns}}(T)$ :

$$
\mathcal{L}_{\Theta}^{\prime}[d-1]=T_{\Theta^{\prime}} P\left(G^{\prime}\right) \times\{0\},
$$

where 0 belongs to $\prod_{v_{i} \in V^{*}} T_{\theta_{j}} \mathrm{SO}(3)$.

We fix in the sequel a configuration $\Theta \in P_{\mathrm{ns}}(T)$. For each $v_{i} \in V^{*}$, we let $W_{i}$ be the subspace of $T_{\Theta} P$ defined as follows:

$$
W_{i}:=\left\{\left[\theta_{1} A_{1} ; \cdots ; \theta_{N} A_{N}\right] \mid A_{k}=0 \text { if } k \neq i, A_{i} \in \mathfrak{s o}(3)\right\} .
$$

Let $v_{j}$ be the unique in-neighbor of $v_{i}$ within $T$. We show below that there exist Lie products $h_{a}$, for $a=1,2,3$, in $\mathcal{L}_{\Theta}^{\prime}[d-1]$ such that

$$
\operatorname{Span}\left\{g_{i j}(\Theta),\left\{\left[g_{i j}, h_{a}\right](\Theta) \mid a=1,2,3\right\}\right\}=W_{i} .
$$

Note that if (22) holds for all $v_{i} \in V^{*}$, then $\mathcal{L}_{\Theta}^{\prime}[d-1]$ and the $W_{i}$, for $v_{i} \in V^{*}$, span the tangent space of $P$ at $\Theta$.

To construct the Lie products $h_{a}$, we let $\phi_{i j}=\theta_{i}^{\top} \theta_{j}$ be the relative attitude and decompose it as $\phi_{i j}:=\psi^{\top} R_{\alpha} \psi$. Because $\Theta$ is nonsingular, $\alpha \neq \pi$. We next define three skew-symmetric matrices as follows:

$$
\tilde{\Omega}_{a}:=\psi^{\top} \Omega_{a} \psi, \quad \forall a=1,2,3,
$$

where $\Omega_{a}$ is defined in (7). Because the matrices $\Omega_{a}$ span $\mathfrak{s o}(3)$ and because the map $\Omega \mapsto \psi^{\top} \Omega \psi$ is a linear automorphism from $\mathfrak{s o}(3)$ to itself, the matrices $\tilde{\Omega}_{a}$ span $\mathfrak{s o}(3)$ as well.

By the spanning condition (21), there exist $h_{a}$, for $a=1,2,3$, in $\mathcal{L}^{\prime}[d-1]$ with the $j$ th component at $\Theta$ given by $\theta_{j} \tilde{\Omega}_{a}$. Note that each $h_{a}$ satisfies the hypothesis of Lemma 5, namely, $h_{a}$ does not depend on $\theta_{i}$ and the $i$ th component of $h_{a}$ is identically zero.

By the definition (20) of $g_{i j}$ and by Lemma 5, the following condition holds for the four vector fields $g_{i j}$ and $\left[g_{i j}, h_{a}\right]$ (for $a=1,2,3$ ): all but their $i$ th components are zero. Moreover, their $i$ th components are given by

$$
\theta_{i}\left(\phi_{i j}-\phi_{j i}\right) \text { and } \theta_{i}\left(\phi_{i j} \tilde{\Omega}_{a}+\tilde{\Omega}_{a} \phi_{j i}\right)
$$

respectively. It remains to show that

$\operatorname{Span}\left\{\phi_{i j}-\phi_{j i},\left\{\phi_{i j} \tilde{\Omega}_{a}+\tilde{\Omega}_{a} \phi_{j i} \mid a=1,2,3\right\}\right\}=\mathfrak{s o}(3)$.

By direct computation,

$$
\left\{\begin{array}{l}
\phi_{i j}-\phi_{j i}=-2 \sin (\alpha) \tilde{\Omega}_{1}, \\
\phi_{i j} \tilde{\Omega}_{1}+\tilde{\Omega}_{1} \phi_{j i}=2 \cos (\alpha) \tilde{\Omega}_{1}, \\
\phi_{i j} \tilde{\Omega}_{2}+\tilde{\Omega}_{2} \phi_{j i}=(1+\cos (\alpha)) \tilde{\Omega}_{2}+\sin (\alpha) \tilde{\Omega}_{3}, \\
\phi_{i j} \tilde{\Omega}_{3}+\tilde{\Omega}_{3} \phi_{j i}=(1+\cos (\alpha)) \tilde{\Omega}_{3}-\sin (\alpha) \tilde{\Omega}_{2} .
\end{array}\right.
$$

Because $\alpha \neq \pi$, every $\tilde{\Omega}_{a}$, for $a=1,2,3$, can be written as a linear combination of the above four matrices. Since the matrices $\tilde{\Omega}_{a}$, for $a=1,2,3$, span $\mathfrak{s o}(3)$, we conclude that (23) holds. Thus,

$$
\mathcal{L}[d]=\mathcal{L}^{\prime}[d-1]+\sum_{v_{i} \in V^{*}} W_{i}=T_{\Theta} P .
$$

This completes the proof.

Remark 1. In the above computation (24), the condition that $\alpha \neq \pi$ is also necessary for the four matrices to span $\mathfrak{s o}(3)$. Indeed, if $\alpha=\pi$, then only the second expression of (24) is nonzero. In this case, by Lemma 4 , the relative attitude $\phi_{i j}$ belongs to $\Phi$ and the configuration $\Theta$ is singular. Note that the above arguments do not imply that the $L A R C$ fails at singular configurations. Instead, they imply that Lie products of greater depths are needed for the $L A R C$ to hold at the singular configurations.

\section{Proof of Theorem 1}

In this section, we show that system (14) is controllable everywhere. We have already shown that the system is controllable over the set of nonsingular configurations $P_{n s}$; the gap we need to bridge is the case where either the initial configuration $\Theta(0)$ or the target $\hat{\Theta}$ is singular.

The proof will be carried out by induction on the number of nodes $N$ in the formation system. We also provide examples to illustrate the key idea of the proof at the end of the section. For the base case where $N=1$, there is only one agent, the leader. Since its dynamics (8) are fully actuated, the system is controllable over $P=\mathrm{SO}(3)$. For the inductive step, we assume that the result holds for $(N-1)$ and prove for $N$.

Let the initial and the target configurations be given by

$$
\Theta(0)=\left[\theta_{1}(0) ; \cdots ; \theta_{N}(0)\right] \text { and } \hat{\Theta}=\left[\hat{\theta}_{1} ; \cdots ; \hat{\theta}_{N}\right],
$$

respectively. We exhibit below control inputs $u_{i j}:[0, \tau] \rightarrow \mathbb{R}$, for $v_{j} v_{i} \in E$, that steer the formation system (14) from $\Theta(0)$ to $\Theta(\tau)=\hat{\Theta}$. To construct the control inputs, we divide the time interval $[0, \tau]$ into three subintervals $[0, \tau / 3)$, $[\tau / 3,2 \tau / 3)$, and $[2 \tau / 3, \tau]$. We construct the control inputs for 
each subinterval and specify the solution $\Theta(t)$ generated by the system (14) at $t_{1}=\tau / 3$ and $t_{2}=2 \tau / 3$.

We again let $T$ be a directed spanning tree of $G$ with $v_{1}$ the root. Without loss of generality, we assume that $v_{N}$ is a leaf of $T$. Let $G^{\prime}=\left(V^{\prime}, E^{\prime}\right)$ be the subgraph of $G$ induced by the first $(N-1)$ nodes, i.e., $V^{\prime}:=\left\{v_{1}, \ldots, v_{N-1}\right\}$. Then, $G^{\prime}$ is rooted with $v_{1}$ a root. Consider the subformation system formed by the first $(N-1)$ agents, with $G^{\prime}$ the underlying digraph. The initial configuration of the subformation system is given by

$$
\Theta^{\prime}(0):=\left[\theta_{1}(0) ; \cdots ; \theta_{N-1}(0)\right] \in P\left(G^{\prime}\right) .
$$

By the induction hypothesis, the subformation system is controllable over the entire configuration space $P\left(G^{\prime}\right)$. Thus, there exist control inputs $u_{i j}:[0, \tau / 3) \rightarrow \mathbb{R}$, for $v_{j} v_{i} \in E^{\prime}$, to steer the subformation system from $\Theta^{\prime}(0)$ to

$$
\Theta^{\prime}(\tau / 3)=\left[\theta_{N}(0) ; \cdots ; \theta_{N}(0)\right] \in P\left(G^{\prime}\right),
$$

i.e., the state for every agent $v_{i}$, for $v_{i} \in V^{\prime}$, is the same as the initial condition $\theta_{N}(0)$ of agent $v_{N}$. Note that any edge $v_{i} v_{N}$ is not in $E^{\prime}$ since $v_{N}$ is not in $V^{\prime}$. We let the corresponding control inputs $u_{N i}$ be identically zero over the period $[0, \tau / 3)$. Then, by construction of the control inputs, the solution $\Theta(t)$ of the complete formation system satisfies

$$
\Theta(\tau / 3)=\left[\theta_{N}(0) ; \cdots ; \theta_{N}(0)\right] \in P(G) .
$$

We next construct the control inputs over the period $[\tau / 3,2 \tau / 3)$. The final configuration $\Theta(\tau / 3)$ of the previous subinterval now becomes the initial condition. Note that $\Theta(\tau / 3)$ is nonsingular because the relative attitudes between the agents are $I \notin \Phi$. By Prop. 3, the formation system (14) is controllable over $P_{\mathrm{ns}}(T)$. Thus, there exist control inputs $u_{i j}:[\tau / 3,2 \tau / 3) \rightarrow \mathbb{R}$, with $v_{j} v_{i} \in E$, such that the solution $\Theta(t)$ generated by the system satisfies

$$
\Theta(2 \tau / 3)=\left[\hat{\theta}_{N} ; \cdots ; \hat{\theta}_{N}\right] \in P_{\mathrm{ns}}(T) .
$$

Note that by $t_{2}=2 \tau / 3$, agent $v_{N}$ reaches its target state $\hat{\theta}_{N}$.

To steer the formation system (14) to reach the target $\hat{\Theta}$, it now suffices steer the subformation system from

$$
\Theta^{\prime}(2 \tau / 3)=\left[\hat{\theta}_{N} ; \cdots ; \hat{\theta}_{N}\right] \in P\left(G^{\prime}\right)
$$

to the target

$$
\hat{\Theta}^{\prime}:=\left[\hat{\theta}_{1} ; \cdots ; \hat{\theta}_{N-1}\right] .
$$

This is feasible by the induction hypothesis. We let $u_{i j}:[2 \tau / 3, \tau] \rightarrow \mathbb{R}$, for $v_{j} v_{i} \in E^{\prime}$, be the control inputs that accomplish the task. The proof is then completed by setting the other control inputs $u_{N i}$, for $v_{i} v_{N} \in E$, identically to zero over the last period $[2 \tau / 3, \tau]$.

Illustration. We first consider a simple formation system composed of only two agents, namely a leader $v_{1}$ and a single follower $v_{2}$. Let the initial and target configurations be

$$
\Theta(0)=[I ; \operatorname{diag}(-1,1,-1)] \text { and } \hat{\Theta}=[I ; \operatorname{diag}(-1,-1,1)] \text {, }
$$

both of which are singular. We illustrate in Fig. 3 how to steer from $\Theta(0)$ to $\Theta(\tau)=\hat{\Theta}$.

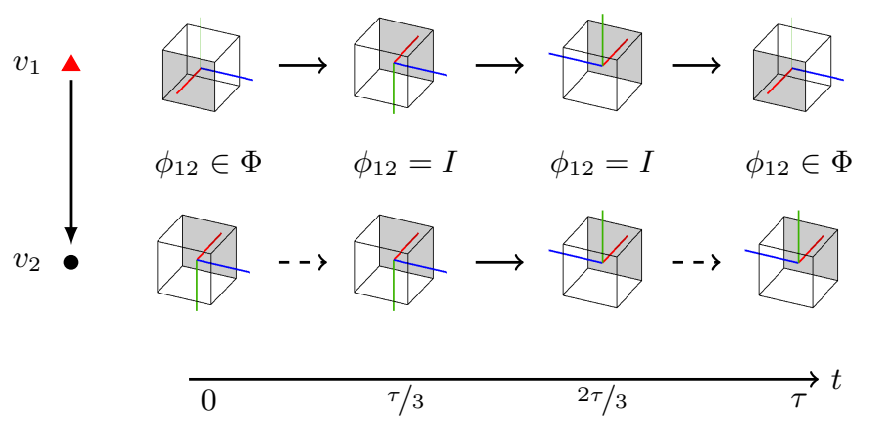

Fig. 3: Simple formation system comprised of a leader and a single follower. We illustrate how to steer the system from one singular configuration to another. The arrows indicate the maneuvers of the agents along the time axis. A dashed arrow indicates that the corresponding agent is stationary in the subinterval. The relative attitudes $\phi_{12}$ between agents $v_{1}$ and $v_{2}$ at $\tau / 3$ and $2 \tau / 3$ are the identity matrix.

The steering process has three steps. 1) Since the leader $v_{1}$ is fully actuated, one can steer $v_{1}$ from $\theta_{1}(0)=I$ to $\theta_{1}(\tau / 3)=\theta_{2}(0)$. The follower $v_{2}$ remains stationary along the process. By doing so, we have a nonsingular configuration $\Theta(\tau / 3)=\left[\theta_{2}(0) ; \theta_{2}(0)\right]$ at time $\tau / 3$. This step can be omitted if the initial configuration $\Theta(0)$ is itself non-singular. 2) Because the set of nonsingular configurations is open, dense, and path connected and because the formation system is controllable over the set of nonsingular configurations, by Prop. 3, one is able to steer from the nonsingular configuration $\Theta(\tau / 3)$ to another nonsingular one $\Theta(2 \tau / 3)=\left[\hat{\theta}_{2}, \hat{\theta}_{2}\right]$. Note that agent $v_{2}$ achieves its target attitude $\hat{\theta}_{2}$ at the end of this step, and will remain stationary in the remainder of the steering process. 3) Finally, one steers the leader $v_{1}$ (fully actuated) from $\theta_{1}(2 \tau / 3)$ to its own target attitude $\hat{\theta}_{1}$ at time $\tau$.

Next, to illustrate the inductive step of the proof, we consider a formation system of 7 agents with the underlying graph shown in Fig. 4a (reproduced from Fig. 1). The graph $G$ is rooted with $v_{1}$ as the single root. The solid arrows indicate a directed spanning tree $T$ of $G$, with $v_{7}$ a leaf. We illustrate in Fig. $4 \mathrm{~b}$ how to steer agent $v_{7}$ from its initial attitude $\theta_{7}(0)$ to its target $\hat{\theta}_{7}$ at time $2 \tau / 3$. Consider the path $v_{1}, v_{2}, v_{5}, v_{7}$ from the root $v_{1}$ to $v_{7}$. The steering process for agent $v_{7}$ involves only the agents in the path and has two steps. 1) By the induction hypothesis, the subformation system formed by agents $v_{1}, v_{2}$, and $v_{5}$ is completely controllable. Thus, one can steer each of these three from its initial attitude to $\theta_{7}(0)$ at time $\tau / 3$. By doing so, the configuration formed by agents $v_{1}, v_{2}, v_{5}$, and $v_{7}$ is nonsingular because all the relative attitudes are the identity matrix. 2) One is then able to steer the subformation system formed by these four agents from $\left[\theta_{7}(0) ; \theta_{7}(0) ; \theta_{7}(0) ; \theta_{7}(0)\right]$ at time $\tau / 3$ to another nonsingular configuration $\left[\hat{\theta}_{7} ; \hat{\theta}_{7} ; \hat{\theta}_{7} ; \hat{\theta}_{7}\right]$ at time $2 \tau / 3$.

The agent $v_{7}$ will then remain stationary in the remainder of the steering process (i.e., the control inputs corresponding to the edges incident to $v_{7}$ will be identically zero over $[2 \tau / 3, \tau]$ ). Note that the subgraph $G^{\prime}$ induced by $v_{1}, \ldots, v_{6}$ is rooted, with one node less than $G$. Thus, by the induction hypothesis, one is able to steer the subformation system corresponding to $G^{\prime}$ from $\Theta^{\prime}(2 \tau / 3)$ to the desired target $\hat{\Theta}^{\prime}$ at time $\tau$. 


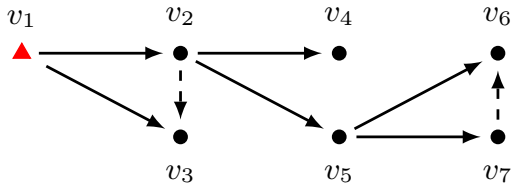

(a) Graph of a formation system. Solid arrows indicate a spanning tree.

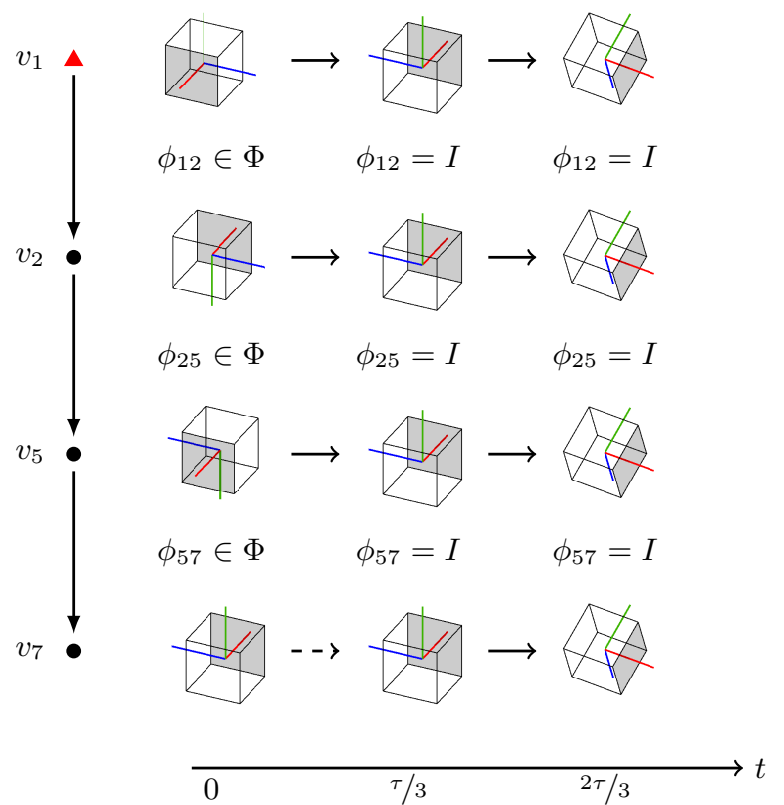

(b) Maneuvers taken by agents $v_{1}, v_{2}, v_{5}$, and $v_{7}$ to steer agent $v_{7}$ from its initial attitude at $t=0$ to the target $\theta_{7}$ at $t=2 \tau / 3$.

Fig. 4: Illustration of the inductive step in the proof of Theorem 1.

Finally, we note that the steering processes described in the above examples are by no means optimal with respect to a certain metric. Rather, they demonstrate the key idea behind the proof of Theorem 1 .

\section{CONCLUSIONS}

Gradient flows have widely been used in formation control to provide a decentralized solution for stabilizing agents at a target configuration. Often, the resulting dynamics possess multiple stable, but undesired, equilibrium points. These equilibrium points are problematic because the associated basins of attraction are open subsets of the configuration space. Thus, the nominal gradient flow can readily enter an undesired basin of attraction, precluding the system from reaching the desired equilibrium. This is especially likely if the target configuration is not close to the starting point. To resolve this issue, we have considered the problem of steering the formation system by manipulating, on the fly, the tuning parameters of the potential functions. Treating these parameters as control inputs, we investigate the controllability problem for a formation system over special orthogonal groups. We have shown that, with some mild assumptions about the underlying information flow topology and the availability of a single fully actuated agent, the formation system is controllable over the entire configuration space.
The proof of the result relies on introducing the nonsingular configurations. We have shown that these configurations form an open, dense, and path-connected subset of the configuration space. We have computed Lie brackets of control vector field and shown that the LARC is satisfied over the nonsingular configurations. Moreover, we have shown that the number of iterative brackets needed for the LARC is bounded above by the depth of the underlying graph (more precisely, any of its directed spanning trees). Finally, the extension of controllability to the entire configuration space (including the singular configurations) has been made. The analysis carried out in the proof provides insights for constructing explicit control laws for steering the formation system, which we will aim to address in future work. There are a few other extensions of the work that we consider interesting for future research. First, we will aim to extend the results to the case where the formation system has multiple leaders, but with the underlying graph being only weakly connected. We will also aim to address the case where the kinematic control model of the individual agents is replaced by other types of system dynamics. Furthermore, we will aim to address the feasibility of reducing the number of control inputs in a formation system to a single one that is common to all agents.

\section{REFERENCES}

[1] L. Krick, M. E. Broucke, and B. A. Francis, "Stabilisation of infinitesimally rigid formations of multi-robot networks," International Journal of Control, vol. 82, no. 3, pp. 423-439, 2009.

[2] S. Mou, M.-A. Belabbas, A. S. Morse, Z. Sun, and B. D. O. Anderson, "Undirected rigid formations are problematic," IEEE Transactions on Automatic Control, vol. 61, no. 10, pp. 2821-2836, 2015.

[3] X. Chen, M.-A. Belabbas, and T. Başar, "Global stabilization of triangulated formations," SIAM Journal on Control and Optimization, vol. 55, no. 1, pp. 172-199, 2017.

[4] K.-K. Oh, M.-C. Park, and H.-S. Ahn, "A survey of multi-agent formation control," Automatica, vol. 53, pp. 424-440, 2015.

[5] M. A. Belabbas, "On global stability of planar formations," IEEE Transactions on Automatic Control, vol. 58, no. 8, pp. 2148-2153, 2013.

[6] U. Helmke and B. D. O. Anderson, "Equivariant Morse theory and formation control," in Annual Allerton Conference on Communication, Control, and Computing (Allerton), pp. 1576-1583, IEEE, 2013.

[7] B. D. O. Anderson and U. Helmke, "Counting critical formations on a line," SIAM Journal on Control and Optimization, vol. 52, no. 1, pp. 219-242, 2014.

[8] S. Zhao and D. Zelazo, "Bearing rigidity theory and its applications for control and estimation of network systems: Life beyond distance rigidity," IEEE Control Systems Magazine, vol. 39, no. 2, pp. 66-83, 2019.

[9] G. Jing, G. Zhang, H. W. J. Lee, and L. Wang, "Angle-based shape determination theory of planar graphs with application to formation stabilization," Automatica, vol. 105, pp. 117-129, 2019.

[10] J. Hu, P. Bhowmick, and A. Lanzon, "Distributed adaptive time-varying group formation tracking for multiagent systems with multiple leaders on directed graphs," IEEE Transactions on Control of Network Systems, vol. 7, no. 1, pp. 140-150, 2019.

[11] S. Zuo, Y. Song, F. L. Lewis, and A. Davoudi, "Time-varying output formation containment of general linear homogeneous and heterogeneous multiagent systems," IEEE Transactions on Control of Network Systems, vol. 6, no. 2, pp. 537-548, 2018.

[12] R. W. Brockett, "System theory on group manifolds and coset spaces," SIAM Journal on control, vol. 10, no. 2, pp. 265-284, 1972.

[13] X. Chen and R. W. Brockett, "Centralized and decentralized formation control with controllable interaction laws," in Proc. IEEE Conference on Decision and Control, pp. 601-606, 2014.

[14] X. Chen, M.-A. Belabbas, and T. Başar, "Controllability of formations over directed time-varying graphs," IEEE Transactions on Control of Network Systems, vol. 4, no. 3, pp. 407-416, 2015. 
[15] B. D. Tapley, S. Bettadpur, M. Watkins, and C. Reigber, "The gravity recovery and climate experiment: Mission overview and early results," Geophysical Research Letters, vol. 31, no. 9, 2004.

[16] S. D'Amico and J. R. Carpenter, "Satellite formation-flying and rendezvous," in Global Positioning System: Theory and Applications, 2017.

[17] W. J. Blackwell, S. Braun, R. Bennartz, C. Velden, M. DeMaria, R. Atlas, J. Dunion, F. Marks, R. Rogers, B. Annane, and R. V. Leslie, "An overview of the TROPICS NASA earth venture mission," Quarterly Journal of the Royal Meteorological Society, vol. 144, pp. 16-26, 2018.

[18] A. Sarlette and R. Sepulchre, "Consensus optimization on manifolds," SIAM Journal on Control and Optimization, vol. 48, no. 1, pp. 56-76, 2009.

[19] M. Lohe, "Non-Abelian Kuramoto models and synchronization," Journal of Physics A: Mathematical and Theoretical, vol. 42, no. 39(395101), 2009.

[20] M. Lohe, "Quantum synchronization over quantum networks," Journal of Physics A: Mathematical and Theoretical, vol. 43, no. 46(465301), 2010.

[21] Y. Kuramoto, "Self-entrainment of a population of coupled non-linear oscillators," in International Symposium on Mathematical Problems in Theoretical Physics, pp. 420-422, Springer, 1975.

[22] J. Markdahl, J. Thunberg, and J. Goncalves, "High-dimensional $\mathrm{Ku}-$ ramoto models on Stiefel manifolds synchronize complex networks almost globally," Automatica, vol. 113(108736), 2020.

[23] L. DeVille, "Synchronization and stability for quantum Kuramoto," Journal of Statistical Physics, vol. 174, no. 1, pp. 160-187, 2019.

[24] J. N. Tsitsiklis, Problems in decentralized decision making and computation. PhD thesis, Dep. Elect. Eng. Comput. Sci., MIT, Cambridge, MA, USA, 1984.

[25] A. Jadbabaie, J. Lin, and A. S. Morse, "Coordination of groups of mobile autonomous agents using nearest neighbor rules," IEEE Transactions on Automatic Control, vol. 48, no. 6, pp. 988-1001, 2003.

[26] R. Olfati-Saber and R. M. Murray, "Consensus problems in networks of agents with switching topology and time-delays," IEEE Transactions on Automatic Control, vol. 49, no. 9, pp. 1520-1533, 2004.

[27] L. Moreau, "Stability of multiagent systems with time-dependent communication links," IEEE Transactions on Automatic Control, vol. 50, no. 2, pp. 169-182, 2005 .

[28] W. Ren and R. W. Beard, "Consensus seeking in multiagent systems under dynamically changing interaction topologies," IEEE Transactions on Automatic Control, vol. 50, no. 5, pp. 655-661, 2005.

[29] J. M. Hendrickx and J. N. Tsitsiklis, "Convergence of type-symmetric and cut-balanced consensus seeking systems," IEEE Transactions on Automatic Control, vol. 58, no. 1, pp. 214-218, 2012.

[30] R. Hermann and A. Krener, "Nonlinear controllability and observability," IEEE Transactions on Automatic Control, vol. 22, no. 5, pp. 728-740, 1977.

[31] V. Jurdjevic, Geometric Control Theory. Cambridge University Press, 1997.

[32] B. Palais and R. Palais, "Eulers fixed point theorem: The axis of a rotation," Journal of Fixed Point Theory and Applications, vol. 2, no. 2, pp. 215-220, 2007.

[33] H. Whitney, "Analytic extensions of differentiable functions defined in closed sets," Transactions of the American Mathematical Society, vol. 36, no. 1, pp. 63-89, 1934.

\section{APPENDIX A}

\section{PROOF OF LEMMA 5}

The vector fields $h$ and $g_{i j}$ are defined on $P=\mathrm{SO}(3)^{N}$. Each $\mathrm{SO}(3)$ is a closed subset of $\mathbb{R}^{3 \times 3}$, so $P$ is a closed subset of $Q:=\left(\mathbb{R}^{3 \times 3}\right)^{N}$. To compute the Lie bracket

$$
\left[g_{i j}, h\right](\Theta)=\frac{\partial h}{\partial \Theta}\left(g_{i j}(\Theta)\right)-\frac{\partial g_{i j}}{\partial \Theta}(h(\Theta)),
$$

we first appeal to the Whitney extension theorem [33] and extend $h$ and $g_{i j}$ so that they are now defined over the entire Euclidean space $Q$ (the extensions are made such that their values at $\Theta \in P$ do not change). With slight abuse of notation, we will still use $h$ and $g_{i j}$ to denote the extended vector fields.
We consider the extension of $g_{i j}$ to take the same form as (20), i.e., for any $\Theta=\left[\theta_{1} ; \cdots ; \theta_{N}\right] \in Q$, we let $g_{i j}$ be defined by

the $k$ th component of $g_{i j}(\Theta):=$

$$
\begin{cases}\theta_{i}\left(\phi_{i j}-\phi_{j i}\right), & \text { if } v_{k}=v_{i} \\ 0, & \text { otherwise }\end{cases}
$$

where $\phi_{i j}:=\theta_{i}^{\top} \theta_{j}$ is defined as usual, but $\theta_{i}$ and $\theta_{j}$ are not necessarily in $\mathrm{SO}(3)$ anymore. For the extension of $h$, we only require that $h$ satisfies the hypothesis of Lemma 5 , i.e., $h$ does not depend on $\theta_{i}$ and the $i$ th component of $h$ is identically zero.

With the above extensions, we will now compute the Lie bracket (A.1). For the first term, we have that

$$
\frac{\partial h}{\partial \Theta}\left(g_{i j}(\Theta)\right)=\lim _{\epsilon \rightarrow 0} \frac{h\left(\Theta+\epsilon g_{i j}(\Theta)\right)-h(\Theta)}{\epsilon} .
$$

Note that the only nonzero component $g_{i j}$ is the $i$ th component, whereas $h$ does not depend on the $i$ th component of its argument. Thus,

$$
h\left(\Theta+\epsilon g_{i j}(\Theta)\right)=h(\Theta)
$$

for all $\epsilon \in \mathbb{R}$, which means that $\frac{\partial h}{\partial \Theta}\left(g_{i j}(\Theta)\right)=0$.

For the second term in (A.1), we have

$$
\frac{\partial g_{i j}}{\partial \Theta}(h(\Theta))=\lim _{\epsilon \rightarrow 0} \frac{g_{i j}(\Theta+\epsilon h(\Theta))-g_{i j}(\Theta)}{\epsilon} .
$$

Because the $k$ th component of $g_{i j}$ is zero, for $v_{k} \neq v_{i}$, we have that the $k$ th component of $\frac{\partial g_{i j}}{\partial \Theta} h(\Theta)$ is 0 for all $v_{k} \neq v_{i}$. It remains to compute the $i$ th component of $\frac{\partial g_{i j}}{\partial \Theta}(h(\Theta))$. Recall that the $k$ th component, for any $v_{k} \in V$, of $h$ at $\Theta \in P$ is given by $\theta_{k} A_{k}(\Theta)$, with $A_{k}(\Theta) \in \mathfrak{s o}(3)$. Then,

$$
\begin{aligned}
& \text { the } i \text { th component of } \frac{\partial g_{i j}}{\partial \Theta}(h(\Theta))= \\
& \qquad \lim _{\epsilon \rightarrow 0} \frac{\tilde{\theta}_{i}(\epsilon)\left(\tilde{\phi}_{i j}(\epsilon)-\tilde{\phi}_{j i}(\epsilon)\right)-\theta_{i}\left(\phi_{i j}-\phi_{j i}\right)}{\epsilon},
\end{aligned}
$$

where $\tilde{\theta}_{i}(\epsilon):=\theta_{i}\left(I+\epsilon A_{i}(\Theta)\right)$ and $\tilde{\phi}_{i j}(\epsilon):=\tilde{\theta}_{i}^{\top} \tilde{\theta}_{j}(\epsilon)$. However, by the hypothesis of Lemma $5, A_{i}(\Theta)=0$, so $\tilde{\theta}_{i}$ and $\tilde{\phi}_{i j}$ reduce to $\tilde{\theta}_{i}=\theta_{i}$ and $\tilde{\phi}_{i j}=\theta_{i}^{\top} \tilde{\theta}_{j}(\epsilon)$, respectively. By computation, the above limit is then given by

$$
\begin{aligned}
\lim _{\epsilon \rightarrow 0} \frac{\theta_{i}\left(\tilde{\phi}_{i j}(\epsilon)-\tilde{\phi}_{j i}(\epsilon)\right)-\theta_{i}\left(\phi_{i j}-\phi_{j i}\right)}{\epsilon \quad \theta_{i}\left(\phi_{i j} A_{j}(\Theta)+A_{j}(\Theta) \phi_{j i}\right),}
\end{aligned}
$$

which completes the proof.

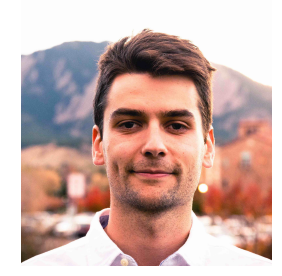

Michael (Misha) Sinner is a doctoral student in the Department of Electrical, Computer and Energy Engineering at the University of Colorado Boulder. $\mathrm{He}$ received the B.Eng. (honors) degree in Mechanical Engineering from the University of Auckland, New Zealand in 2015 and the M.S. degree in Electrical, Computer, and Energy Engineering from the University of Colorado Boulder in 2018. His research interests include control theory, optimization, estimation, distributed control, and their applications, particularly in the field of renewable energy. 


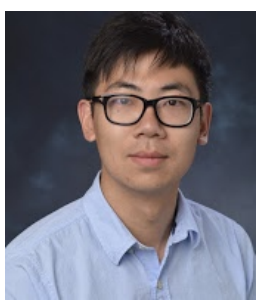

Xudong Chen is an Assistant Professor in the Department of Electrical, Computer, and Energy Engineering at the University of Colorado Boulder. Previously, he was a postdoctoral fellow in the Coordinated Science Laboratory at the University of Illinois, Urbana-Champaign. He obtained the B.S. degree from Tsinghua University, Beijing, China, in 2009, and the Ph.D. degree in Electrical Engineering from Harvard University, Cambridge, Massachusetts, in 2014. He was an awardee of the 2020 Air Force Young Investigator Program. His research interests are in the area of control theory, stochastic processes, optimization, game theory and their applications in modeling, analysis, control, and estimation of large-scale complex systems.

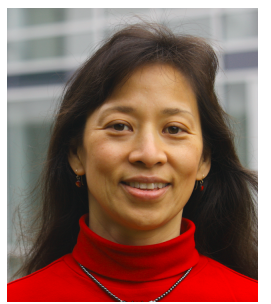

Lucy Pao is the Palmer Endowed Chair Professor in the Electrical, Computer, and Energy Engineering Department at the University of Colorado Boulder. She earned B.S., M.S., and Ph.D. degrees in Electrical Engineering from Stanford University. Her research has primarily focused on engineering control systems, with applications ranging from atomic force microscopes to multi-megawatt wind energy systems. She is a Fellow of the IEEE and IFAC. Selected recent awards include the 2012 IEEE Control Systems Magazine Outstanding Paper Award (with K. Johnson), the 2015 Society for Industrial and Applied Mathematics (SIAM) Journal on Control and Optimization Best Paper Prize (with J. Marden and H. P. Young), the 2017 Control Engineering Practice Award from the American Automatic Control Council, the Scientific Award 2017 from the European Academy of Wind Energy, and the 2019 Nyquist Lecturer Award from the ASME Dynamic Systems \& Control Division. Selected recent and current professional society activities include being a Fellow of the Renewable and Sustainable Energy Institute (2009-present), General Chair of the 2013 American Control Conference, member of the IEEE Control Systems Society (CSS) Board of Governors (2011-2013 and 2015), IEEE CSS Fellow Nominations Chair (2016-2018), member of the IFAC Fellow Selection Committee (2014-2017 and 2017-2020), and member of the IFAC Executive Board (2017-2020). 